\title{
Clinical results of endoscopic sciatic nerve decompression for deep gluteal syndrome: mean 2-year follow-up
}

\author{
Myung-sik Park, Sun-Jung Yoon*, Sung-yeop Jung and Seung-Ho Kim
}

\begin{abstract}
Background: The purpose of this study is to assess the effectiveness of endoscopic sciatic nerve decompression and evaluated the differences of clinical results between atraumatic and traumatic groups.

Methods: Sixty consecutive patients. We retrospectively reviewed sixty consecutive patients without major trauma (45 hips) or with major trauma (15 hips) groups to compare the outcomes of endoscopic treatment.). The mean follow-up period was $24 \pm 2.6$ months (range, 24-38.4 months).

Results: The mean duration of symptoms was 14.1 months (range, 12 to 32 months). Compromising structures were piriformis muscle, fibrovascular bundles, and adhesion with scar tissues. The mean VAS score for pain decreased from $7.4 \pm 1.5$ to $2.6 \pm 1.5(P=.001)$. The mean $\mathrm{mHHS}$ increased from $81.7 \pm 9.6$ to $91.8 \pm 7.6(P=.003)$. Clinically, positive paresthesia and seated piriformis test were statistically significant to diagnosis sciatic entrapment syndrome. Paresthesia and sitting pain were significantly improved at the final follow-up $(P=.002)$. More favorable outcome was observed a group without major trauma. No complication was observed.

Conclusions: Endoscopic sciatic nerve decompression is a safe and effective procedure for the management of DGS. Patients with major trauma could have poor clinical outcome. Seated piriformis test, FADIR, and tenderness of sciatic notch are maybe useful guide for pre and postoperative evaluation of DGS.
\end{abstract}

Keywords: Endoscopic sciatic nerve decompression, Sciatic nerve entrapment, Deep gluteal syndrome

\section{Background}

Deep gluteal syndrome (DGS) involves pain in the buttock caused from entrapment of the sciatic nerve in deep gluteal space [1]. The boundaries of deep gluteal space are femoral neck anteriorly, gluteus maximus posteriorly, linea aspera of proximal femur laterally, sacrotuberous ligament medially, inferior margin of the sciatic notch superiorly and hamstring muscle inferiorly (Fig. 1). The space contains piriformis, obturator internus/externus, gemelli, quadratus femoris, hamstring, gluteal nerves and lateral ascending vessels of the medial femoral circumflex artery. Any contents of deep gluteal space can cause sciatic nerve entrapment syndrome [2]. Posttraumatic DGS can be occurred by

\footnotetext{
*Correspondence: sjyoon_kos@naver.com

Department of Orthopedic Surgery, Research Institute of Clinical Medicine of Chonbuk National University- Biomedical Research Institute of Chonbuk National University Hospital, Jeonju, South Korea
}

scarring around the piriformis muscle and the sciatic nerve as well as the adherence of the nerve to the posterior pelvic coulum [3].

Patients presenting with sciatic nerve entrapment often complain symptoms of sitting pain (inability to sit for $30 \mathrm{~min}$ ), walking pain, radicular pain of the lower back or hip, and paresthesia of the affected buttock and inguinal area $[4,5]$. Diagnosis can made by several physical examinations include tenderness on sciatic notch, Flexion-ADduction-Internal Rotation (FADIR) test, Pace sign [6], Lasègue test and seated piriformis test $[2,4,7]$. A variant of the Freiberg test involves flexion, adduction, and internal rotation of the hip [3, 8]. The Pace sign is pain and weakness with resisted abduction and external rotation of the hip [6]. The Lasègue sign is pain with straight leg raise testing (to $90^{\circ}$ hip flexion) $[9,10]$. Usually, magnetic resonance arthrography (MRA) and electromyography (EMG) usually don't provide proper 


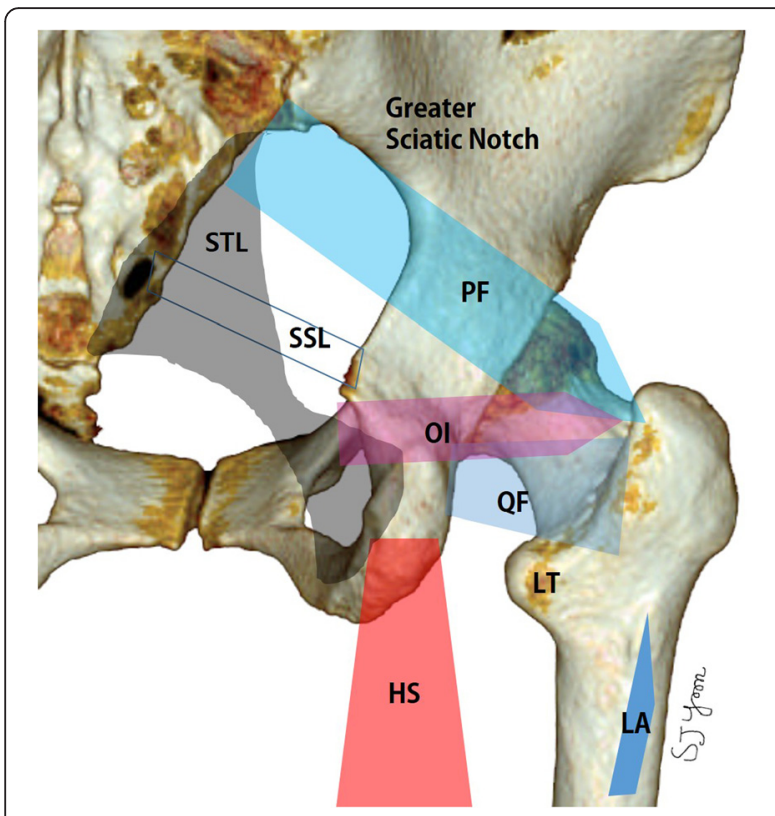

Fig. 1 Schematic image of the deep gluteal space, HS, hamstring origin; $L A$, linea aspera; LT, lesser trochanter; Ol, obturator internus; PF, piriformis; SSL, sacrospinous ligament; QF, Quadratus Femoris

information for DGS. So, clinical symptoms and physical examinations are essential for diagnosis of sciatic nerve entrapment syndrome [2]. In the patients of DGS who have moderate to severe symptoms or undergo failure of conservative treatment, sciatic nerve decompression through open or endoscopic technique can be performed for release of pain $[2,4,7]$.

There are difficulties to diagnose by one of clinical presentations, physical examinations, or objective evaluations. Moreover, it still remains unclear which symptoms or signs of this complex syndrome would be resolved after endoscopic decompression. To our knowledge, this is the first study regarding differences between atraumatic and traumatic deep gluteal syndrome.

The purpose of this study is to assess 1) which clinical presentations or physical examinations will improve after the endoscopic sciatic nerve decompression 2) the effectiveness of this endoscopic procedure, 3) differences of clinical outcome between groups associated with or without major trauma.

\section{Methods}

Endoscopic sciatic nerve release was performed from September 2009 to June 2013. This study was approved by institutional review board of the Chonbuk National University Hospital. Total of 66 patients who had endoscopic sciatic nerve decompression for sciatic nerve entrapment syndrome with a minimum 24 months follows up were included in this study. Three patients excluded, who had taken total hip arthroplasty, two patients suffered from direct nerve injury after acetabular fracture and one patient with severe spondylosis. The rest 60 patients were included in this study. There were 33 males and 27 females with a mean age of $48.60 \pm$ 11.46 years (range, 20-70 years). The mean follow-up period was $24 \pm 2.6$ months (range, 24-38.4 months). The mean duration of symptoms was $10.6 \pm 0.9$ months (range, 4-30 months). A comprehensive back and hip physical examination ruled out the spine disease and sacroiliac joint disease. Our treatment protocol is shown in Fig. 2.

The clinical diagnosis of the DGS has been advocated by patient history, clinical presentations and physical examinations. We applied described clinical symptoms and physical examinations to elicit the posterior pain according to Martin et al [2]. Clinical presentations included 1) walking pain, 2) sitting pain (in ability to sit for $>30 \mathrm{~min}$ ), 3) radicular pain and 4) paresthesia. The physical examinations included 1) tenderness on sciatic notch, 2) FADIR test, 3) Pace sign, 4) Lasègue test and 5) seated piriformis test. A test was considered positive withthe recreation of the posterior pain [8].

The anteroposterior pelvic radiograph and MRA were made with the patient supine on the table with both lower extremities oriented in $20^{\circ}$ of internal rotation using a boots in order to maximize the length of the femoral neck. All radiographic images were acquired digitally through a Picture Archiving and Communication System (PACS, Marotech, Maroview, Seoul).

The radiologic measurements such as the neck-shaft angle (NSA), lateral center-edge (LCE) angle on pelvis $\mathrm{AP}$ radiographs and femoral, acetabular anteversion and ischiofemoral space (IF space) on a MRA were assessed for all patients $[11,12]$. The measuring of the ischiofemoral space is the smallest distance between the lateral cortex of the ischial tuberosity and medial cortex of the lesser trochanter, and quadratus femoris space. All MRA examinations were evaluated for qualitative change by consensus by one musculoskeletal radiologist and two orthopedic surgery fellows. We also check the electromyography for the symptoms of radicular pain in eight patients selectively to differentiate other compressive neuropathies of sciatic nerve.

Surgical procedure was done with a supine position on the hip arthroscopic table. Diagnostic hip arthroscopy was performed for all patients with deep gluteal syndrome. If the patient had definitive intraarticular lesion under hip arthroscopy, the lesion would be tried to treat especially if their posterior hip pain has alleviated by intra-articular hip injection test. Then the sciatic nerve was explored. For approach to the deep gluteal area, we make a posterolateral and/or axillary portals. The 


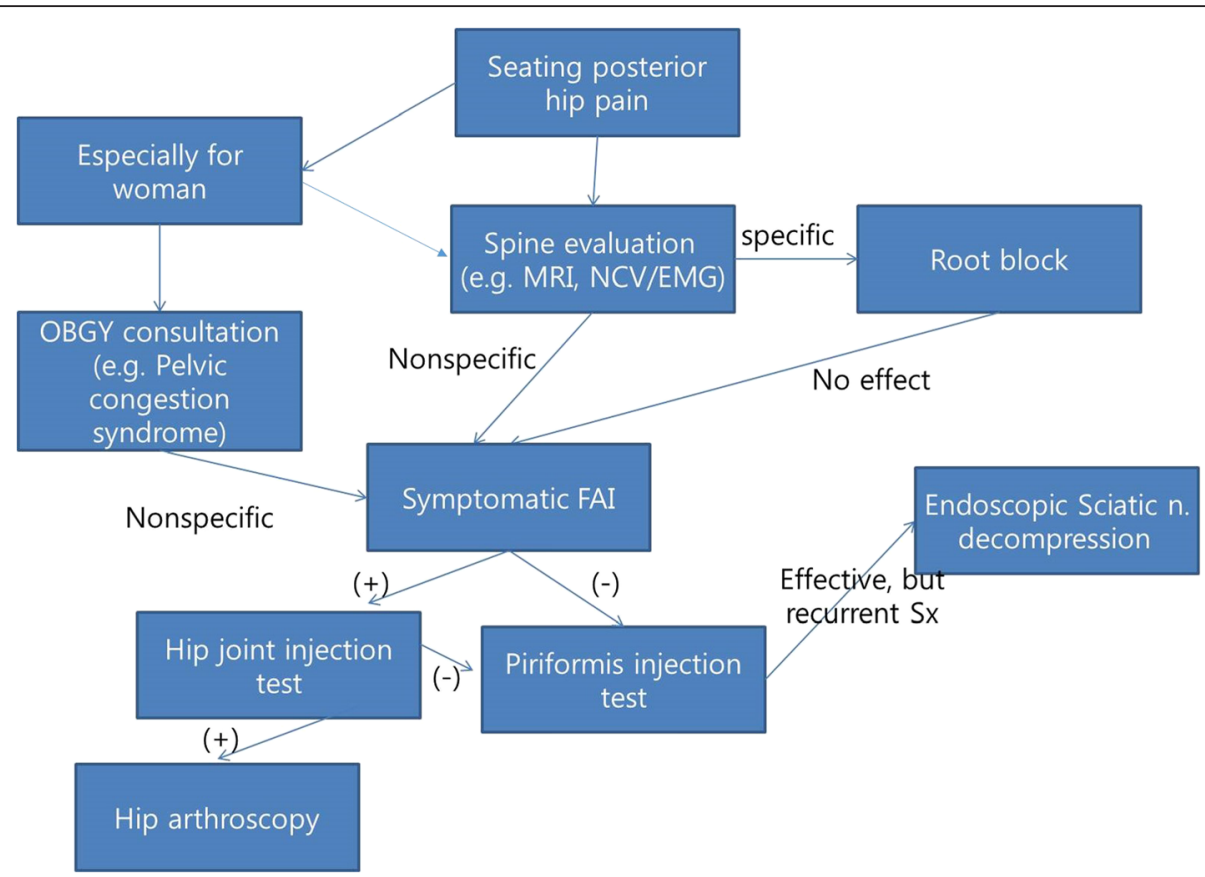

Fig. 2 Flow-chart showing treatment process. A flow-chart showing the decision making process for endoscopic sciatic nerve decompression of seating posterior hip pain

axillary portal is placed $3-4 \mathrm{~cm}$ superior or inferior to center between anterolateral and posterolateral portals. Through the endoscopy, sciatic nerve was detected, that passes around piriformis, obturator internus and gemelli muscles in deep gluteal space (Fig. 3). Sciatic nerve release was performed by removal of fibrovascular scar bands or splitting tendinous portion of piriformis, obturator internus, and quadriceps femoris muscles and or bony spicules which compromised sciatic nerve excursion [2]. The fibrovascular scars were delicately cauterized by use of a radiofrequency probe and removed by use of

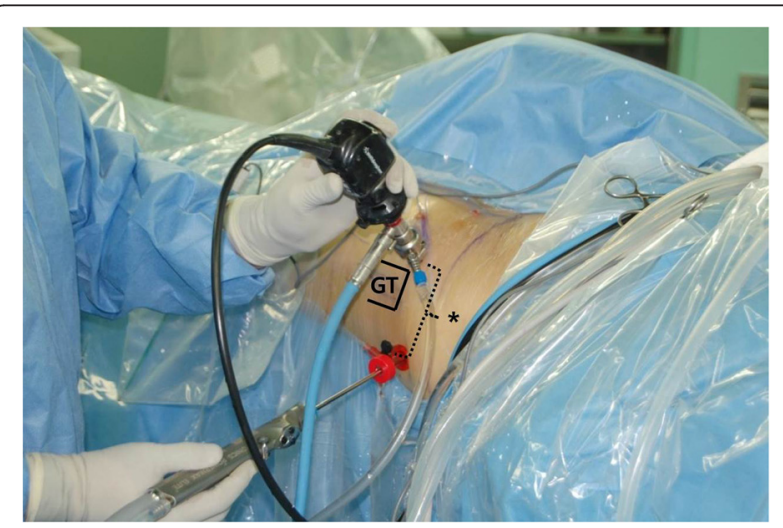

Fig. 3 Endoscopic portals for sciatic nerve decompression. The anterolateral portal placement is one $\mathrm{cm}$ anterolateral to the anterior corner of the greater trochanter (GT). The posterolateral portal is four-fingerbreadth posterior to the anterior portal* arthroscopic shaver or dissection scissors. After release, using nerve retractor, we identified improvement of tightness and gliding of the sciatic nerve while internal and external rotation or flexion and extension of hip repeatedly [13]. Postoperative one day, we allowed partial weight bearing with crutch and full weight bearing at three weeks.

Clinical outcomes was assessed by persistence or not of the symptoms and physical examinations, VAS score, modified Harris Hip Score (mHHS) [14], Hip outcomes score (HOS daily life), SF-12 (12-Item Short Form Survey) and modified Benson surgical outcomes rating [3] which divided as poor, fair, good, and excellent (Table 1). To compare the outcomes of endoscopic sciatic nerve decompression between fracture and nonfracture patients, we divided two groups as like who had not taken any pelvic or acetabular surgery group (45 patients) and associated prior pelvic or acetabular fracture surgery group, 15 patients. Of these patients, acetabular fracture in 11 patients and associated with pelvic ring fracture in four patient.

Statistical analyses were performed using a SPSS (version 8.0). Categorical variables are reported as frequencies and percentage and compared between preoperative and postoperative results with Fisher's exact tests. Continuous variables are summarized as means and SDs and compared between groups with independent-samples $t$ tests. All statistical test were 2 -sided, and $P$ values $<.05$ were considered statistically significant. 
Table 1 Benson surgical outcomes rating

\begin{tabular}{ll}
\hline Outcomes & Symptoms \\
\hline Excellent & No pain with prolonged periods of sitting ( $>30 \mathrm{~min})$, strenuous activity, bending, twisting, stairs, rapid walking, jogging. \\
Good & No pain with short periods of sitting ( $\leq 30 \mathrm{~min})$ or daily activities or mild pain with prolonged periods of sitting or strenuous activity. \\
Fair & Occasional mild pain with short periods of sitting or normal daily activities or moderate pain with prolonged sitting or strenuous activity. \\
Poor & Severe pain with short periods of sitting or normal daily activities, little change from preoperative level of pain associated with sciatic nerve. \\
\hline
\end{tabular}

\section{Results}

On plain pelvis, preoperative NSA was $131.9 \pm 4.7^{\circ}$, and LCE angle was $29.8 \pm 5.4^{\circ}$. Using with a MRA femoral anteversion $15.9 \pm 9.9^{\circ}$, acetabular anteversion $13.8 \pm 5.6^{\circ}$ and ischiofemoral space was $13.4 \pm 3.8 \mathrm{~mm}$ in lesion site and $15.1 \pm 4.4 \mathrm{~mm}$ in contralateral side $(P=.000)$ was measured (Table 2). Ninety percent of patient complaining sit pain was improved after surgery. Preoperative symptoms of 1) sit pain (inability to sit for more than $30 \mathrm{~min}), 2$ ) paresthesia, 3) radicular pain, and 4) walking pain were improved $90.9 \%(P=.002), 88.8 \%, 45.3 \%$, and, $50 \%$ respectively at 2 year follow-up. Preoperative sitting pain and paresthesia were significantly improved after endoscopic decompression.

For physical examination, $90 \%$ of patients showing positive seated piriformis test was disappeared of sign after surgery. Preoperative positive physical examination of 1) tenderness on sciatic notch, 2) FADIR test 3) Pace sign, 4) Lasègue test, and 5) seated piriformis test were improved $90.7 \%, 70.2 \%, 66.6 \%, 65.3 \%$, and $64.3 \%$, respectively at two year follow-up. Preoperative sciatic notch pain, FADIR test, and Seated piriformis test were significantly improved after sciatic nerve decompression $(P<.001)$. Postoperative clinical symptoms improvement and positive physical examination were shown Table 2.

During surgery, we found the operative findings compatible with femoroacetabular impingements in 27 hips included 7 hips as a mixed type, 12 hips as a cam type, and 8 hips as a pincer type. Arthroscopic FAI surgery was underwent for only symptomatic FAI (16 of 27

Table 2 Clinical presentations and physical examinations

\begin{tabular}{llll}
\hline Clinical presentations & Preop & Postop & $P$ \\
\hline Walking pain & $11(18.3 \%)$ & $6(10 \%)$ & 0.240 \\
$\begin{array}{l}\text { Sit pain (inability to sit for } \\
\text { more than } 30 \text { min) }\end{array}$ & $53(88.3 \%)$ & $5(8.3 \%)$ & 0.000 \\
Radicular pain & $12(20 \%)$ & $6(10 \%)$ & 0.130 \\
Paresthesia & $36(60 \%)$ & $4(6.7 \%)$ & 0.000 \\
Physical exam & & & \\
Tenderness & $42(70 \%)$ & $14(23.3 \%)$ & 0.000 \\
FADIR & $26(43.3 \%)$ & $9(15 \%)$ & 0.000 \\
Pace'sign & $14(23.3 \%)$ & $5(8.3 \%)$ & 0.060 \\
Lasègue & $10(16.7 \%)$ & $3(5.0 \%)$ & 0.070 \\
Seated piriformis & $43(71.7 \%)$ & $4(6.7 \%)$ & 0.000 \\
\hline
\end{tabular}

patients) proven by preoperative evaluation including intra-articular injection test.

In deep gluteal space, as anatomical etiologies of sciatic nerve entrapment, the fibrovascular scar bands were observed in 27 hips (Table 3), variation of the tendinous portion of piriformis muscle in 25 hips, variation of the obturator internus in four hips, hypertrophy of the quadratus femoris muscles in three hips and thickened bursa extending from greater trochanter in three hips (Fig. 4), heterotopic ossification around fracture site in one hips, and simple fibrous band dissection in seven hips.

The mean of ischiofemoral space in DGS groups are $13.6 \pm 3.8 \mathrm{~mm}$ (range, 4.1-21.4) and contralateral side are $15.2 \pm 4.5 \mathrm{~mm}$ (range, 4.1-26.9) $(p=0.000)$. And other radiologic markers were described (Table 4).

Postoperatively, patient satisfaction was evaluated as excellent in 15, good in 38 , and fair in seven patients. There were no postoperative complications as like compartment syndrome or sciatic nerve injuries. Patient satisfaction in trauma group was statistically lower than it in disease group.

\section{Discussion}

Deep gluteal syndrome is a condition in which the sciatic nerve is compressed by any structures in deep gluteal space. The chief symptom were caused by sciatic nerve entrapment surrounding structures which usually causes buttock pain and a complained of the inability to sit for a long period of time. Pain became worse during sitting, walking and flexion movement combined with internal rotation of the hip. And also patients may complain radicular pain of affected leg, much like nerve root pain associated with lumbar disc disease [4]. Previously as the term "piriformis syndrome" that implies a type of deep gluteal syndrome [15]. However, any structures in

Table 3 Caused structures of sciatic nerve entrapment

\begin{tabular}{ll}
\hline Compromising structures & Number of hips $(n=60)$ \\
\hline $\begin{array}{l}\text { Fibrous scar bands } \\
\text { with Gluteus maximus bursa (3) }\end{array}$ & 27 \\
Piriformis muscle and triceps coxae & 25 \\
$\quad$ with Obturator internus tendon (4) & \\
$\quad$ with Quadratus femoris muscles (3) & \\
Vascular compression & 7 \\
Heterotopic ossification & 1 \\
\hline
\end{tabular}




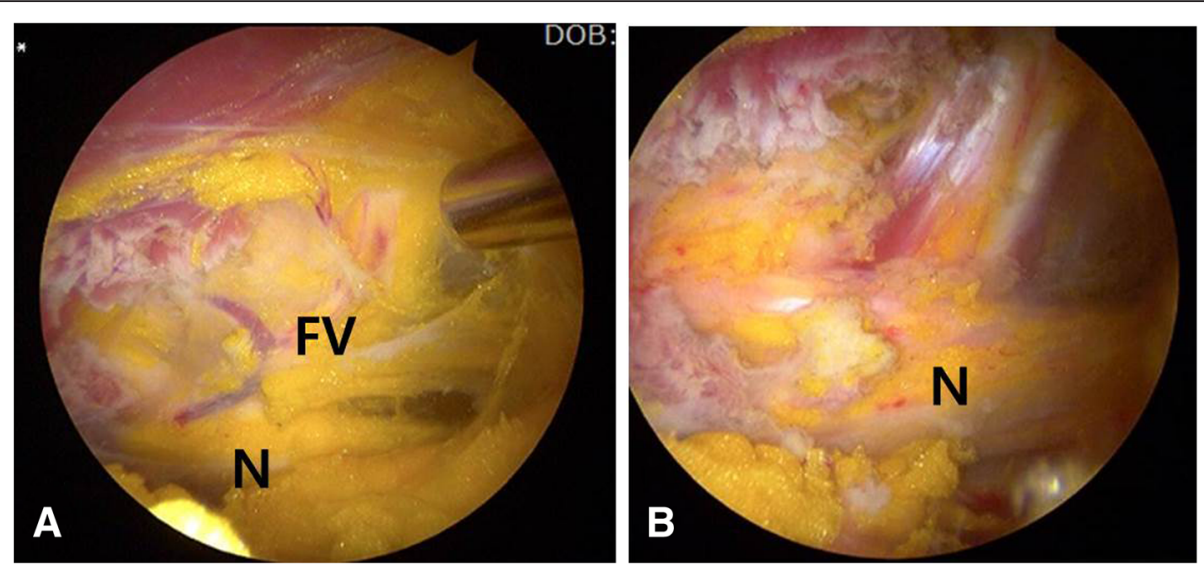

Fig. 4 Sciatic nerve entrapment by fibrovascular bands. a Endoscopic view of sciatic nerve (N) by fibrovascular bands (FV). b Endoscopic view of sciatic nerve $(\mathrm{N})$ after decompression

deep gluteal region can cause this syndrome by compression sciatic nerve, so sciatic nerve entrapment syndrome or deep gluteal syndrome may be a proper representation [16]. There are a number of etiological factors of sciatic nerve entrapment syndrome, such as direct trauma of buttock or pelvis, hypertrophy of muscles in deep gluteal region, hematoma or neoplasm and anatomical variants between piriformis and sciatic nerve, etc $[17-19]$. Be aware of possible anatomical variation bifurcation of the sciatic nerve through the muscles. In this study, fibrous scar band and anatomical variation of tendinous portion of the piriformis were most frequent intraoperative findings. Otherwise, intraneural and perineural fibrosis was the most common arthroscopic finding in major trauma group. The prevalence of the piriformis and sciatic nerve anomaly in piriformis syndrome patients has been reported by others [20]. It was not significantly different from what is thought to be a normal population, it indicated that this anomaly may not be as important in the pathogenesis of piriformis syndrome as previously thought.

In this study, all patients were evaluated with physical examinations, routine pelvis radiography, and also MRA. We couldn't find a specific feature of DGS in radiologic variables such as, neck-shaft angle, femoral anteversion and acetabular anteversion in this study. However, by previous study, in some positions that cause sciatic nerve compression, such as the FADIR test, electromyography may have greater specificity and sensitivity than other available tests for the diagnosis of piriformis syndrome [7]. Bryan et al suggested layered concepts of anatomical structure of the hip. The pathologies of osseous and cartilage layers can affected the clinical presentations of musculotendinous or neural layers [18]. In this study, we repaired symptomatic labral lesions and corrected FAI deformity [21].

A high index of suspicion is needed to make this diagnosis of DGS in a patient with posterior hip or leg pain in absence of lumbar pathology. This pain is usually aggravated while sitting with more than $30 \mathrm{~min}$ or no back pain. Sciatic nerve entrapment due to varicose vein or pyomyositis of piriformis muscle in a pediatric patient was also reported [22, 23]. We suggested repeated trauma of the piriformis muscle as sudden stretching of muscle or long-term exercise and traumatic subgluteal muscular damages. These surrounding soft tissue structures and heterotopic ossification sometimes may be act as pressure to the passage of the sciatic nerve into the piriformis muscle and decompression is the last resort.

In this study, tenderness of deep gluteal space, FADIR test, and seated piriformis test showed more statistical

Table 4 Results of imaging study of DGS patients

\begin{tabular}{llll}
\hline Variables & Mean value & & $P$ \\
\hline Neck-shaft angle $\left(^{\circ}\right)$ & $131.9 \pm 4.7$ & $2\left(>140^{\circ}\right)$ & $3\left(<125^{\circ}\right)$ \\
Femoral anteversion $\left(^{\circ}\right)$ & $15.9 \pm 9.9$ & $12\left(20^{\circ}\right)$ & $14\left(<10^{\circ}\right)$ \\
LCE ${ }^{\text {a }}$ angle $\left(^{\circ}\right)$ & $29.8 \pm 5.4$ & $1\left(>42^{\circ}\right)$ & $15\left(<26^{\circ}\right)$ \\
& Ipsilateral side & Contralateral side & .000
\end{tabular}


significance $(P<.001)$ to diagnosis DGS, but Pace sign $(P=.06)$ and Lasègue test has no significance $(P=.070)$.

According to Martin et al., MRI usually doesn't provide sufficient information for DGS [2, 24]. In our study, of 60 patients with sciatic nerve entrapment syndrome, only six patients showed minor swelling of sciatic nerve. Decreased distance of ischiofemoral space have a statistical significance with symptom of DGS $(P<0.001)$, which might be related with ischiofemoral impingement. The diagnostic efficacy of MR neurography has been reported by others [25]. Piriformis muscle asymmetry and sciatic nerve hyperintensity at the sciatic notch exhibited a $93 \%$ specificity and $64 \%$ sensitivity. Cutoff values of ischiofemoral and quadratus femoris space has been propose by Torriani et al. $\leq 17 \mathrm{~mm}$, and $\leq$ $8 \mathrm{~mm}$, subsequently.

Sciatic nerve neuropathy associated fracture or reconstructive surgery of the acetabulum were managed by open method according to Issack et al [5]. After the open decompression, most of sensory symptoms are improved for minimum of one year, but there were no patients with worsening of neurologic examination after release. They reported also improved symptoms of a tenderness and or paresthesia of affected leg after surgery and some improvement of foot drop. However, there are risks associated with open technique such as hematoma, infection, poor cosmesis, and long rehabilitation time [3]. This endoscopic management shows improvement of sitting pain and sensory changes around buttocks and no complications.

This study has several limitations, including a retrospective cohort study, small numbers of case and relatively short follow-up duration. And few patients in this study had endoscopic surgery for sciatic nerve entrapment syndrome and FAI at the same time. For proper evaluation of endoscopic surgery for sciatic nerve entrapment syndrome, additional well-designed prospective studies, in a group of patients with only sciatic nerve entrapment syndrome without intraarticular lesions are necessary.

\section{Conclusion}

Endoscopic sciatic nerve decompression is a safe and effective procedure for the management of DGS. Patients with major trauma could have poor clinical outcome. Seated piriformis test, FADIR, and tenderness of sciatic notch are maybe useful guide for pre and postoperative evaluation of DGS.

\section{Abbreviations}

DGS: deep gluteal syndrome; EMG: electromyography; FADIR: FlexionADduction-Internal Rotation; FAl: femoroacetabular impingement; HOS: hip outcome score; IF: ischiofemoral; LCE: lateral center-edge angle; MHHS: modified Harris hip score; MRA: magnetic resonance arthrography; NSA: neck-shaft angle; VAS: visual analogue scale.

\section{Acknowledgments}

This paper was supported by Fund of Biomedical Research Institute, Chonbuk National University Hospital.

\section{Availability of data and materials}

The data set supporting the conclusion of this article is available on request to the corresponding author.

\section{Authors' contributions}

MSP, SJY, SYJ, and SHK mad substantial contributions to design of the study. SJY was responsible for drafting this manuscript. SYJ performed the statistical analysis. MSP and SJY have given valuable advice and comments on this manuscript. SJY and SHK involved in the analysis and interpretation of data. All authors read and approved the final manuscript.

\section{Competing interests}

The authors declare that they have no competing interests.

\section{Ethics of approval and consent to participate}

This retrospective study was approved by the Institutional Review Board of our hospital, a tertiary referral center for hip and pelvis disease. Informed consent was waived because of the retrospective nature of this study.

Received: 29 December 2015 Accepted: 7 May 2016

Published online: 20 May 2016

\section{References}

1. Martin HD, Palmer IJ. History and physical examination of the hip: the basics. Curr Rev Musculoskelet Med. 2013;6:219-25.

2. Martin HD, Shears SA, Johnson JC, Smathers AM, Palmer IJ. The endoscopic treatment of sciatic nerve entrapment/deep gluteal syndrome. Arthrosc J Arthrosc Relat Surg Off Publ Arthrosc Assoc N Am Int Arthrosc Assoc. 2011; 27:172-81.

3. Benson ER, Schutzer SF. Posttraumatic piriformis syndrome: diagnosis and results of operative treatment. J Bone Joint Surg Am. 1999;81:941-9.

4. Hopayian K, Song F, Riera R, Sambandan S. The clinical features of the piriformis syndrome: a systematic review. Eur Spine J Off Publ Eur Spine Soc Eur Spinal Deform Soc Eur Sect Cerv Spine Res Soc. 2010;19:2095-109.

5. Issack PS, Kreshak J, Klinger CE, Toro JB, Buly RL, Helfet DL. Sciatic nerve release following fracture or reconstructive surgery of the acetabulum. Surgical technique. J Bone Joint Surg Am. 2008;90(Suppl 2 Pt 2):227-37.

6. Pace JB, Nagle D. Piriform syndrome. West J Med. 1976;124:435-9.

7. Boyajian-O'Neill LA, McClain RL, Coleman MK, Thomas PP. Diagnosis and management of piriformis syndrome: an osteopathic approach. J Am Osteopath Assoc. 2008;108:657-64.

8. Papadopoulos EC, Khan SN. Piriformis syndrome and low back pain: a new classification and review of the literature. Orthop Clin North Am. 2004;35:65-71.

9. Freiberg $\mathrm{AH}$. SCiatic pain and its relief by operations on muscle and fascia. Arch Surg. 1937;34:337-50.

10. Freiberg AH, Vinke TH. Sciatica and the Sacro-lliac Joint. J Bone Jt Surg Am. 1934;16:126-36.

11. Clohisy JC, Carlisle JC, Trousdale R, Kim Y-J, Beaule PE, Morgan P, et al. Radiographic evaluation of the hip has limited reliability. Clin Orthop. 2009; 467:666-75.

12. Ali AM, Teh J, Whitwell D, Ostlere $\mathrm{S}$. Ischiofemoral impingement: a retrospective analysis of cases in a specialist orthopaedic centre over a four-year period. Hip Int J Clin Exp Res Hip Pathol Ther. 2013;23:263-8.

13. Coppieters MW, Alshami AM, Babri AS, Souvlis T, Kippers V, Hodges PW. Strain and excursion of the sciatic, tibial, and plantar nerves during a modified straight leg raising test. J Orthop Res Off Publ Orthop Res Soc. 2006;24:1883-9.

14. Byrd JW, Jones KS. Prospective analysis of hip arthroscopy with 2-year follow-up. Arthrosc J Arthrosc Relat Surg Off Publ Arthrosc Assoc N Am Int Arthrosc Assoc. 2000;16:578-87.

15. Grgić V. Piriformis muscle syndrome: etiology, pathogenesis, clinical manifestations, diagnosis, differential diagnosis and therapy. Liječnic ki Vjesn. 2013;135:33-40.

16. McCrory P, Bell S. Nerve entrapment syndromes as a cause of pain in the hip, groin and buttock. Sports Med Auckl NZ. 1999;27:261-74.

17. Beaton LE, Anson BJ. The Sciatic Nerve and the Piriformis Muscle: Their Interrelation a Possible Cause of Coccygodynia. J Bone Jt Surg Am. 1938;20:686-8 
18. Jankovic D, Peng P, van Zundert A. Brief review: piriformis syndrome: etiology, diagnosis, and management. Can J Anaesth J Can Anesth. 2013;60:1003-12.

19. Kitagawa Y, Yokoyama M, Tamai K, Takai S. Chronic expanding hematoma extending over multiple gluteal muscles associated with piriformis syndrome. J Nippon Med Sch Nippon Ika Daigaku Zasshi. 2012;79:478-83.

20. Smoll NR. Variations of the piriformis and sciatic nerve with clinical consequence: a review. Clin Anat N Y N. 2010;23:8-17.

21. Draovitch P, Edelstein J, Kelly BT. The layer concept: utilization in determining the pain generators, pathology and how structure determines treatment. Curr Rev Musculoskelet Med. 2012;5:1-8.

22. Hu M-H, Wu K-W, Jian Y-M, Wang C-T, Wu I-H, Yang S-H. Vascular compression syndrome of sciatic nerve caused by gluteal varicosities. Ann Vasc Surg. 2010; 24:1134. e1-4.

23. Toda T, Koda M, Rokkaku T, Watanabe H, Nakajima A, Yamada T, et al. Sciatica caused by pyomyositis of the piriformis muscle in a pediatric patient. Orthopedics. 2013;36:e257-9.

24. Martin RL, Philippon MJ. Evidence of validity for the hip outcome score in hip arthroscopy. Arthrosc J Arthrosc Relat Surg Off Publ Arthrosc Assoc N Am Int Arthrosc Assoc. 2007;23:822-6.

25. Filler AG, Haynes J, Jordan SE, Prager J, Villablanca JP, Farahani K, et al. Sciatica of nondisc origin and piriformis syndrome: diagnosis by magnetic resonance neurography and interventional magnetic resonance imaging with outcome study of resulting treatment. J Neurosurg Spine. 2005;2:99-115.

\section{Submit your next manuscript to BioMed Central} and we will help you at every step:

- We accept pre-submission inquiries

- Our selector tool helps you to find the most relevant journal

- We provide round the clock customer support

- Convenient online submission

- Thorough peer review

- Inclusion in PubMed and all major indexing services

- Maximum visibility for your research

Submit your manuscript at www.biomedcentral.com/submit

) Biomed Central 